\title{
Novel D-type Fiber Optic Localized Plasmon Resonance Sensor Realized by Femtosecond Laser Engraving
}

\author{
Chien-Hsing Chen ${ }^{1}$, Tzu-Chien Chao ${ }^{2}$, Wan-Yun $\mathrm{Li}^{3}$, Wei-Chih Shen ${ }^{4}$, Chung-Wei Cheng ${ }^{4}$, Jaw-Luen Tang ${ }^{1}$, Lai-Kwan \\ $\mathrm{Chau}^{* 3}$ and Wei-Te $\mathrm{Wu}^{* 5}$ \\ ${ }^{1}$ Department of Physics, National Chung Cheng University, Chia-Yi, Taiwan \\ ${ }^{2}$ Department of Mechanical Engineering, National Chung Cheng University, Chia-Yi, Taiwan \\ ${ }^{* 3}$ Department of Chemistry and Biochemistry, National Chung Cheng University, Chia-Yi, Taiwan \\ E-mail: chelkc@ccu.edu.tw \\ ${ }^{4}$ ITRI South, Industrial Technology Research Institute, Tainan, Taiwan \\ ${ }^{* 5}$ Department of Biomechatronics Engineering, National Pingtung University of Science and Tech- \\ nology, Pingtung, Taiwan \\ E-mail: weite@mail.npust.edu.tw
}

\begin{abstract}
A novel fiber optic localized plasmon resonance (FO-LPR) sensor composed of a D-shape optical fiber was proposed and demonstrated in the present study. The D-shape optical fiber was fabricated by a femtosecond laser micromachining system. The dimensions of the D-shape zone were $100 \mu \mathrm{m}$ in depth measured from the surface of the polymer jacket layer, and the total length was 6 $\mathrm{mm}$. After annealing treatment, the mean roughness of the surface was $231.7 \mathrm{~nm}$ as determined by AFM. The exposed surface of the D-shape fiber was modified with self-assembled gold nanoparticles to produce the FO-LPR sensor. The response of the sensor shows that the signal increases linearly with increasing refractive index. The sensor resolution of the sensor was determined to be $4.1 \times 10^{-4}$ RIU.

DOI: $10.2961 / \mathrm{jlmn} .2010 .01 .0001$
\end{abstract}

Keywords: D-shape optical fiber, femtosecond laser, fiber optic localized plasmon resonance

\section{Introduction}

Biosensors for the diagnosis of diseases, food, and environmental detection of biological agents have increasing demands over the past few decades. Traditionally, labeling of either the substrate or the receptor for the promising detection is often needed. However, the procedures of labeling in general are tedious and sometimes extra errors may be induced. To avoid these problems, the main purpose of the present work is to develop a small-size fiberoptic probe for label-free and real-time chemical and biological detection. A label-free sensing platform based on the modification of the unclad portion of an optical fiber with self-assembled Au colloids as the transducer has been developed by our group [1-2]. The detection scheme is by fiber optic localized plasmon resonance, so called FO-LPR. Colloidal gold exhibits a distinct absorption band when the incident frequency of photon is resonant with the collective oscillation of the conduction electrons, which is known as localized plasmon resonance (LPR) [3-7]. The extinction spectrum of the LPR band is highly dependent upon the local environment of the nanoparticles. The relative low absorbance of a colloidal gold layer can be enhanced by a fiber optic evanescent-wave sensing scheme [1-2]. A 400 $\mu \mathrm{m}$-diameter core plastic-clad silica optical fiber was adopted as the waveguide; therefore, the absorbance can be significantly enhanced [1]. The core of the optical fiber is required to be exposed to reagent solutions for modification with self-assembled colloidal gold monolayer on the unclad portion of an optical fiber [1-2]. Thus, the cladding of the optical fiber was stripped by fire or by $\mathrm{CO}_{2}$ laser machining to expose the fiber core. According to the principle of the detection scheme, using smaller optical fibers with a smaller core diameter will increase the number of total internal reflections per unit length in the optical fiber and, hence, better performance of LPR-based biosensors is expected. Furthermore, it reduces the dimension of the probe as well. To enhance the performance of LPR-based biosensors and to take advantage of the technologies established by the fiber-optic communication industries, the communication grade multimode optical fiber was employed as the waveguide, which was composed of silica based core $(62.5 \mu \mathrm{m}$ in diameter), cladding and polymer jacket with outer diameters of 125 and $250 \mu \mathrm{m}$, respectively. However, there are two issues arisen of using small diameter optical fibers as the waveguide. Since communication grade optical fibers with a certain length of cladding and jacket entirely removed is fragile and easily broken, the first issue is how to maintain enough mechanical strength while part of the cladding and jacket has been removed. In order to overcome this mechanical strength issue, D-shape fibers were fabricated. Another issue is that both the core and cladding layers of communication grade multimode optical fiber are composed of fused silica, and it is a transparent, hard and brittle material which could not be effectively machined by fire or by the continuous long-pulsed laser. The second issue can be overcome by choosing short-pulsed laser, femtosecond laser. The most prominent features of the femtosecond laser compared with conventional continuous longpulsed laser are ultra short pulse duration and very strong instantaneous power. Femtosecond laser can photoinduce 
the non-linear multi-photon absorption of a material during irradiation without creating the laser-induced heat affected zone [8-9]. It can be used to engrave on transparent, hard and brittle materials very precisely without inducing any micro cracks and heat affected zone. This paper demonstrates the possibility of fabricating the D-shape optical fibers and the performance of such a novel FO-LPR probe for refractive index sensing, which is a prerequisite test for label-free bio-detection by the FO-LPR technology.

\section{Experiment section}

\subsection{Preparation of Au nanoparticles}

Aqueous solution of hydrogen tetrachloroaurate $(20 \mathrm{~mL}$, $0.88 \mathrm{mM}$ ) was brought to a vigorous boil and stirred for 10 min. Freshly prepared sodium citrate solution $(2.4 \mathrm{~mL}, 1 \%$ $(\mathrm{w} / \mathrm{v}))$ was rapidly added to the hydrogen tetrachloroaurate solution with vigorous stirring. After the solution was further stirred for $20 \mathrm{~min}$, the color of the solution changed from yellow to deep red. Then the solution was allowed to cool to room temperature with stirring [10].

\subsection{Femtosecond laser micromachining system for manufacturing $D$-shaped fiber optics}

A femtosecond laser micromachining system (as illustrated in Fig. 1) was used for engraving the trench on the optical fiber. The femtosecond laser was a regenerative amplified mode-locked Ti:sapphire laser with pulse duration $\sim 120$ fs after the compressor, central wavelength 800 $\mathrm{nm}$, repetition rate of $1 \mathrm{kHz}$, and maximum pulse energy of $\sim 3.5 \mathrm{~mJ}$. To adjust the energy of the laser beam, the linear polarized Gaussian laser beam from the laser was attenuated by a rotatable half-wave plate and a polarizing beam splitter (PBS). To measure the laser energy, a certain fraction of the laser beam was split off by a beam splitter (BS) and measured with a power detector. The number of laser shots applied to the sample was controlled by an electromechanical shutter. The laser beam was focused onto the fiber by a 10x objective lens (numerical aperture $0.26, \mathrm{M}$ Plan Apo NIR, Mitutoyo) mounted on a Z stage. D-type trench under fabrication was translated by a computer controlled X-Y micro-positioning stage with error less than 1 $\mu \mathrm{m}$. The fabrication process was monitored by a chargecoupled device (CCD).

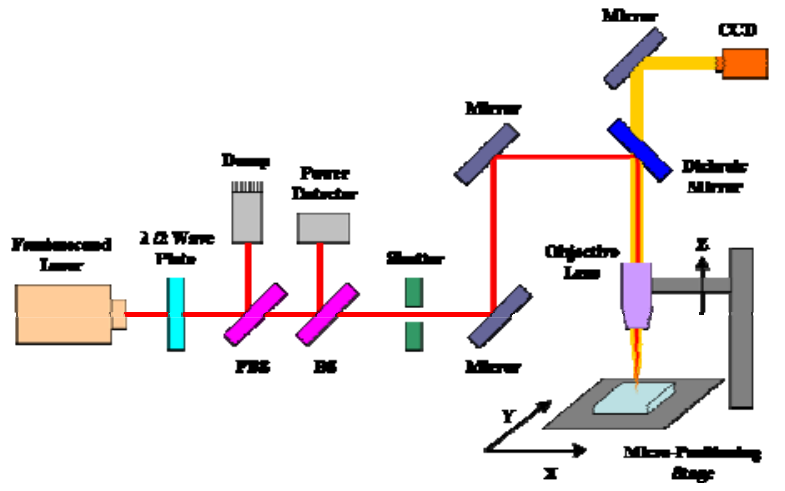

Fig. 1 Experimental setup of femtosecond laser micromachining system for fabrication of D-shape optical fibers.

\subsection{Preparation of gold nanoparticle-modified optical fibers}

For the sensor a 62.5/125 multimode all-silica fiber with a $250 \mu \mathrm{m}$ buffer (Corning) was used. A femtosecond laser micromachining system was used to engrave a Dshape trench on the optical fiber. Because of the negative surface charge of gold nanoparticles, the positive charge of poly(allylamine hydrochloride) can serve as a linker between the negatively charged silica surface and Au nanoparticles [11-12]. As such, the exposed silica surface in the D-shape trench was then modified with poly(allylamine hydrochloride) by immersing the cleaned D-shape optical fibers into vials of $3 \mathrm{mM}$ solution of poly(allylamine hydrochloride). After $15 \mathrm{~min}$, the optical fibers were removed from the solution and rinsed with pure water to remove unbound monomers from the surface. After thorough rinsing, the D-shape optical fibers were immersed into the gold nanoparticles solution (with absorbance of about 1) for 30 min to modify gold nanoparticles on the surface of the Dshape trench. The optical fibers were then rinsed with pure water to remove unbound gold nanoparticles on the surface.

\subsection{Instrumentation and measurements}

Figure 2 is an illustration of the experimental setup for sensing measurements with the gold nanoparticlesmodified D-shape optical fiber. The fiber-optic sensing system used to measure the transmission power of the sensor was consisted of a function generator (model GFG-8255A, Good Will Instrument, Inc.), a LED light source $(\lambda=530$ $\mathrm{nm}$ ), a sensing D-shape fiber modified with Au nanoparticles, a microfluidic chip, a photodiode (model 2001, New Focus, Inc.), a lock-in amplifier (model 7225R, EG\&G Instrument, Inc.) and a computer for data acquisition. Absorption spectra of the samples were obtained by using a HP 8453 spectrophotometer. Transmission electron microscopic (TEM) observations of the samples, which had been dispersed and allowed to dry on copper grids, were taken with a Joel TEM 1200 EX instrument. The images of the D-shape optical fiber and immobilized gold nanoparticles on exposed surface of the D-shape fiber were obtained using a Hitachi 4800I field-emission scanning electron microscope (FE-SEM) operated at $10 \mathrm{keV}$.

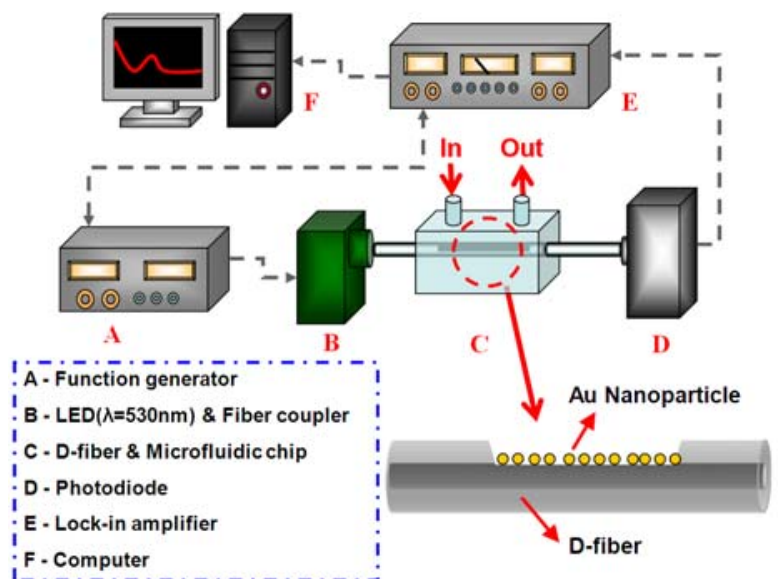

Fig. 2 Schematic representation of the experimental setup used to make sensing measurements with the gold nanoparaticlesmodified D-shape optical fiber.

\section{Results and discussion}




\subsection{Characteristics of Au nanoparticles}

Au nanoparticles were prepared according to the above procedure mentioned in Section 2.1. The absorption peak is $518 \mathrm{~nm}$ as shown in Fig. 3. TEM image is also showed in the inset of Fig. 3. A histogram derived from TEM image analysis shows that the mean diameter of the gold nanoparticles is $13.5 \mathrm{~nm}$.

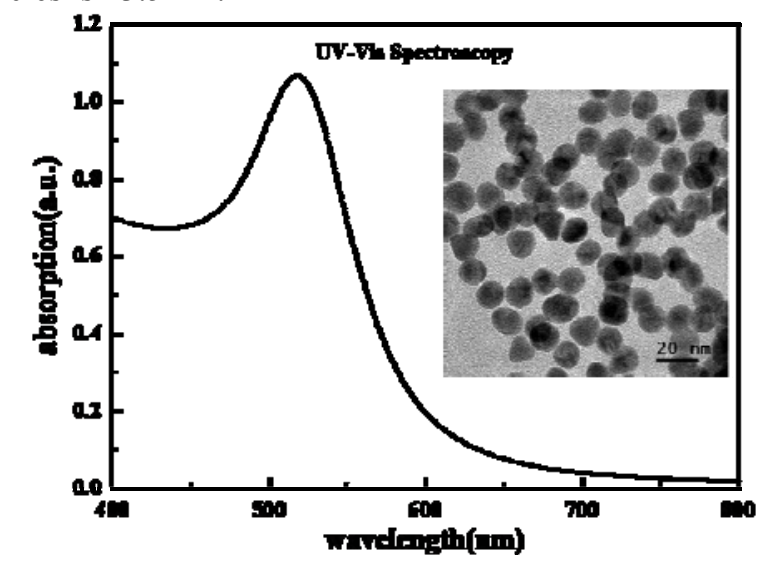

Fig. 3 Absorbance spectra of gold nanoparticles in a pH 7.4 PBS buffer (Inset: TEM image of the nanoparticles).

\subsection{D-shape optical fibers fabricated by femtosecond laser micromachining system}

In laser processing applications, the focus spot size is one of the key parameters of the system. In order to verify the focus spot size of the femtosecond laser micromachining system. A flat fused silica plate (a kind of transparent, hard and brittle material) was used to find out machining parameters. As shown in Fig. 4, the laser pulse energy was adjusted to fabricate a series of holes on the flat fused silica plate. Then, average diameters of these holes under various processing energies were measured. The diameter of the hole increases with increasing laser pulse energy (see Fig. 4). Base on these data, the engraving threshold of the fused silica plate and the focus spot size of the system were calculated by the following equations [8]:

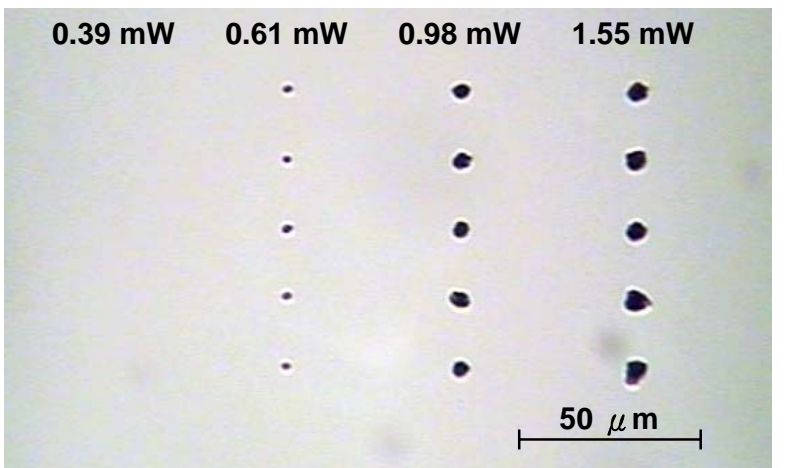

Fig. 4 The optical microscopic image of the flat fused silica plate suffered from different laser fluences.

$$
\begin{aligned}
& F_{0}^{a v}=\frac{E_{\text {pulse }}}{\pi w_{0}^{2}} \\
& D^{2}=2 w_{0}^{2} \ln \left(\frac{F_{0}^{a v}}{F_{t h}}\right)
\end{aligned}
$$

where $\mathrm{F}_{0}{ }^{\mathrm{av}}$ is the laser average fluence $\left(\mathrm{J} / \mathrm{cm}^{2}\right), \mathrm{E}_{\text {pulse }}$ is the laser pulse energy $(\mu \mathrm{J}), \mathrm{w}_{0}$ is focus spot radius $(\mu \mathrm{m}), \mathrm{D}$ is the diameter of the hole $(\mu \mathrm{m})$, and $\mathrm{F}_{\mathrm{th}}$ is the engraving threshold $\left(\mathrm{J} / \mathrm{cm}^{2}\right)$. The relationship between squared diameter of holes and fluence is shown in Fig. 5. The focus spot radius $\left(\mathrm{w}_{0}\right)$ is $2.44 \mu \mathrm{m}$, and the engraving threshold of fused silica plate is $2.68 \mathrm{~J} / \mathrm{cm}^{2}$. According to the above data, the performance of the femtosecond laser micromachining system is suitable for precise machining of communication grade multimode optical fibers.

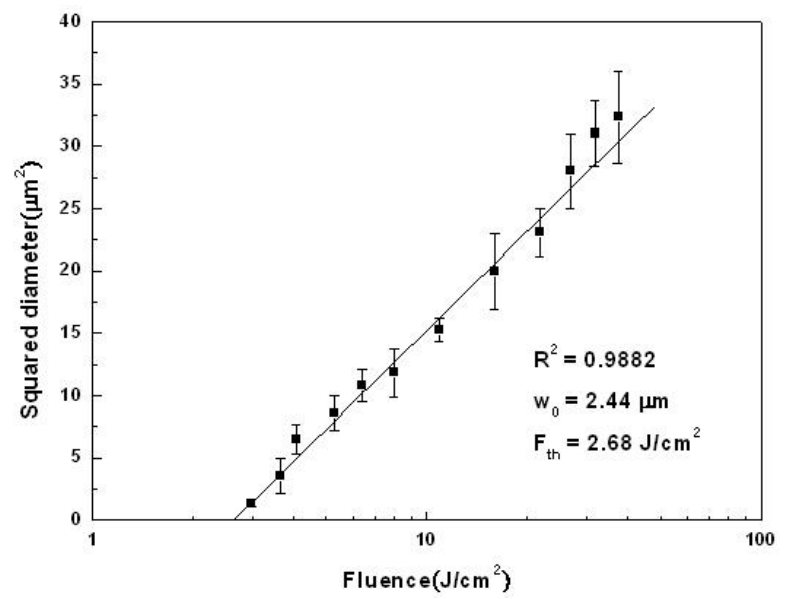

Fig. 5 The relationship between squared diameter of holes on the flat fused silica plate and fluence.

During the fabrication of the D-shape optical fiber, the transmission power was monitored with a fiber optic light source $(\lambda=1550 \mathrm{~nm}$, model MPS-8012, Lightwave, Inc.) and a multifunction optical meter (model AQ2140, ANDO, Inc.). The monitoring experimental step is shown in Fig. 6. The average power loss measured for five samples is 0.75 $\pm 0.33 \mathrm{~dB}\left(\mathrm{~dB} \operatorname{loss}=10 \times \log \mathrm{P}_{2} / \mathrm{P}_{1}, \mathrm{P}_{1}=\right.$ input power, $\mathrm{P}_{2}=$ output power). It can be seen that D-shape optical fiber induces about $16 \%$ transmission power loss keeping enough transmission power for further testing.

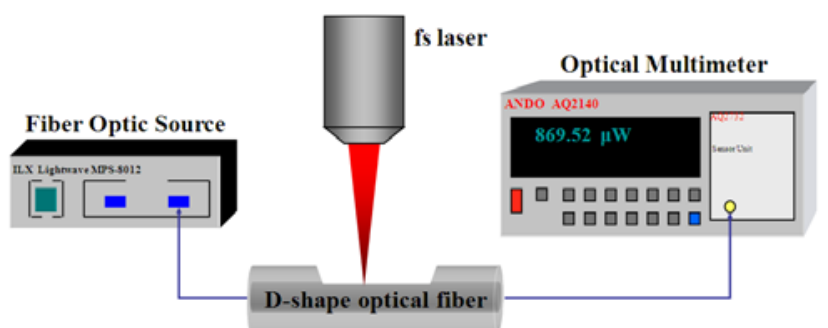

Fig. 6 Experimental setup for monitoring the transmission power loss of D-shape optical fibers during fabrication process.

Fig. 7 shows the illustration diagram of the D-shape optical fiber. The dimensions of the D-shape zone were 100 $\mu \mathrm{m}$ in depth measured from the surface of the polymer jacket layer, and the total length was $6 \mathrm{~mm}$. Fig. 8 shows the SEM image of the D-shape optical fibers fabricated by the femtosecond laser. As shown in Fig. 9, the surface mean roughness is $231.7 \mathrm{~nm}$ as measured by an AFM (model Multi Mode, Veeco, Inc.) 


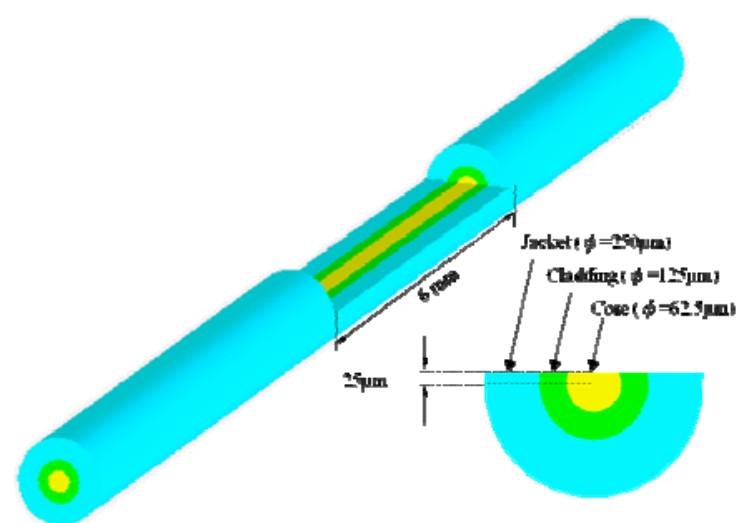

Fig. 7 The illustration diagram of the D-shape optical fiber.

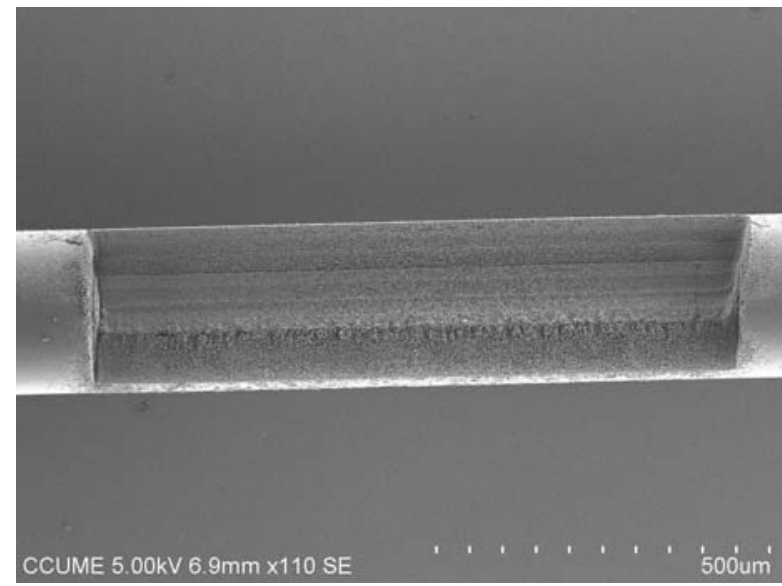

Fig. 8 FE-SEM image of the D-shape optical fiber.

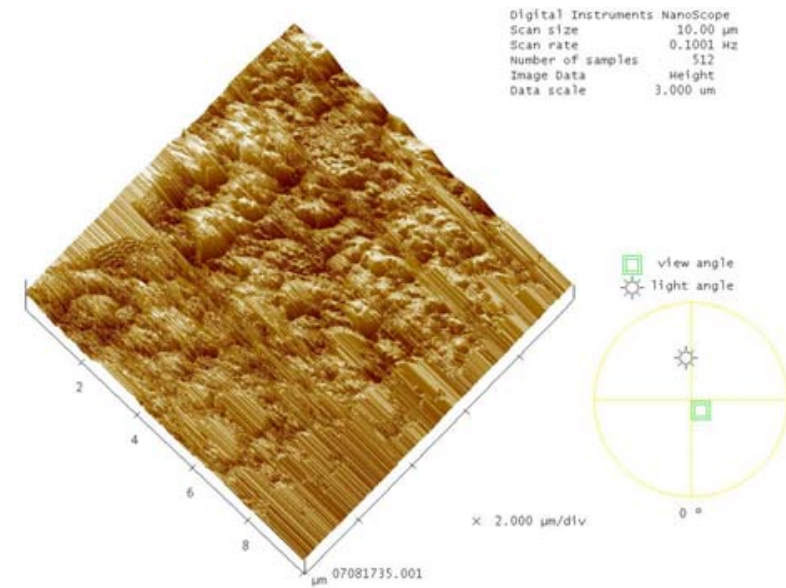

Fig. 9 AFM image of the exposed core surface (scanning area 10 $\mu \mathrm{m} \times 10 \mu \mathrm{m}$.$) .$

\subsection{Gold nanoparticles-modified D-shape optical fiber and sensing test}

The preparation of gold nanoparticles-modified Dshape optical fiber followed the procedure in Section 2.3. The SEM image of the exposed surface after gold nanoparticles-modification is revealed in Fig. 10. Apparently, many Au nanoparticles with uniform distribution are immobilized on the surface. Such D-shape fibers modified with gold nanoparticles on the exposed core surface were used for further sensing test. The ability of the gold nanoparticlesmodified D-shape fiber to detect changes in the surround- ing refractive index was studied. The control of surrounding refractive index was through the use of sucrose solutions with various concentrations [13]. Figure 11 shows a linear fit $(R=0.9983)$ of the plot of the transmission power as a function of the refractive index. The sensor resolution (sensor resolution $=3 \sigma / m, \sigma=$ standard deviation of $I$ in measuring the blank, $m=$ slope) of the sensor was determined to be $4.1 \times 10^{-4}$ RIU.

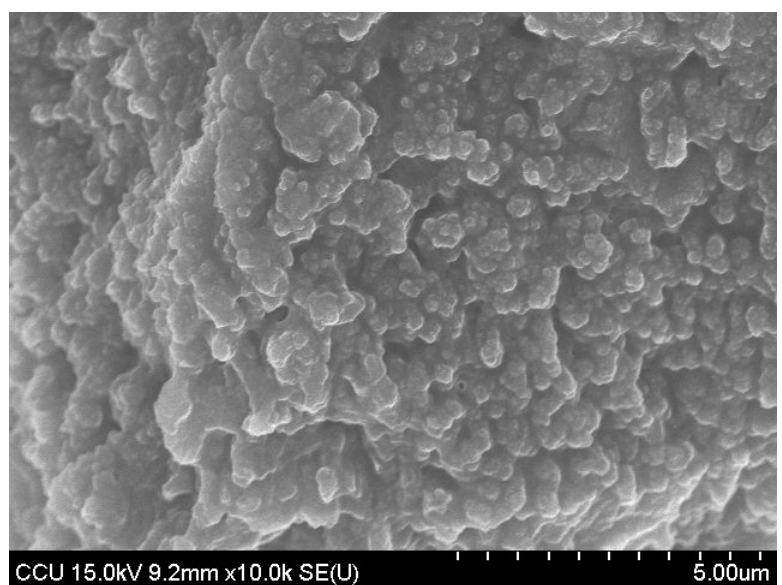

Fig. 10 FE-SEM images of the exposed surface of the D-shape fiber after modification with gold nanoparticles. (zoom in 10k)

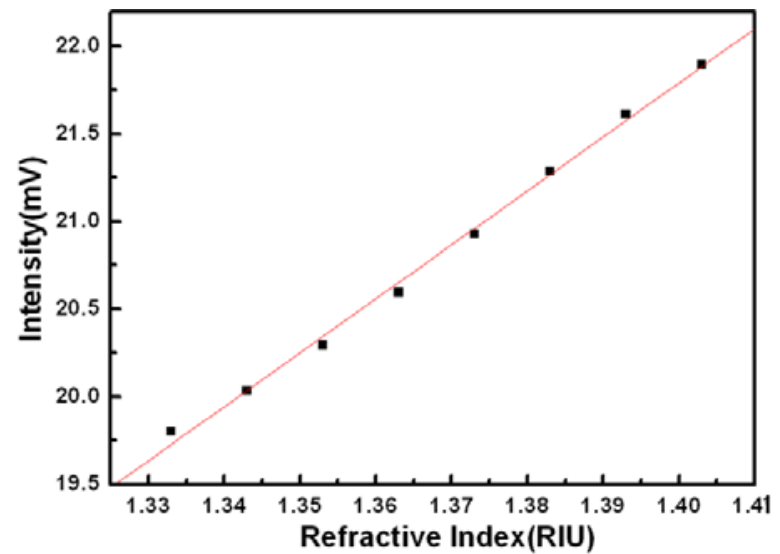

Fig. 11 Plot of the transmission power vs. refractive index of the sucrose solution.

\section{Conclusions}

This study successfully demonstrates the feasibility of fabricating the D-shape optical fibers by the femtosecond laser, and making a transmission-based FO-LPR probe for chemical sensing. With a focus spot radius of the femtosecond laser micromachining system of approximately 2.44 $\mu \mathrm{m}$, the surface roughness of the exposed fiber core was $231.7 \mathrm{~nm}$. The average transmission power loss is $0.75 \mathrm{~dB}$ which is induced by D-shape optical fiber. The sensor resolution of the gold nanoparticles-modified D-shape optical fiber obtained by transmission power interrogation was $4.1 \times 10^{-4}$ RIU.

\section{Acknowledgments}

Support of this research by ITRI South (Taiwan, R.O.C.) through Grant No. B200-97G270001884 and the National Science Council (Taiwan, R.O.C.) through Grant No. NSC 95-3114-P-194-001-MY3 is acknowledged. 


\section{References}

[1] S. F. Cheng and L. K. Chau: Anal. Chem., 75, (2003) 16.

[2] L. K. Chau, Y. F. Lin, S. F. Cheng and T. J. Lin: Sens. actuators. B Chem., 113, (2006) 100.

[3] S. Underwood and P. Mulvaney: Langmuir 10, (1994) 3427.

[4] T. R. Jensen, M. L. Duval, K. L. Kelly, A. A. Lazarides, G. C. Schatz and R. P. Van Duyne: J. Phys. Chem. B, 103, (1999) 9846.

[5] T. Okamoto, I. Yamaguchi and T. Kobayashi: Opt. Lett., 25, (2000) 372.

[6] A. C. Templeton, J. J. Pietron, R. W. Murray and P. Mulvaney: J. Phys. Chem. B, 104, (2000) 564.
[7] N. Nath and A. Chilkoti: Anal. Chem., 74, (2002) 504.

[8] A. Ben-Yakar, R. L. Byer: J. Appl. Physi., 96, (2004) 5316.

[9] R. Gattass and E. Mazur: Nature Photonics, 2, (2008) 219.

[10]N. Nath, A. Chilkoti: Anal. Chem., 76, (2004) 5370.

[11] J. Schmitt, P. Machtle, D. Eck, H. Mohwald and C. A. Helm: Langmuir, 15, (1999) 3256.

[12] J. Bravo, L. Zhai, Z. Wu, R. E. Cohen and M. F. Rubner: Langmuir, 23, (2007) 7293.

[13]Hwang T C (1994): "Experiments in Physical Chemistry”, (Gaulih Book Co., Taipei, 1994).

(Received: July 9, 2009, Accepted: December 2, 2009) 